weight change from age 20 in relation to the risk of CVD mortality, including specific causes of CVD, using data of a large populationbased cohort study in Japan.

Methods In 1994, 41364 eligible individuals aged 40-79years responded to the questionnaire survey. Participants were categorised by weight change: loss $\geq 10 \mathrm{~kg}$ and $5-9.9 \mathrm{~kg}, \pm 4.9 \mathrm{~kg}$, gain $5-9.9 \mathrm{~kg}$ and $\geq 10 \mathrm{~kg}$. During the follow-up period of 13.3 years, 1756 deaths of CVD were identified and risk analysis was done by Cox proportional hazards model.

Results For all CVD mortality in men were significantly increased for weight loss more than $10 \mathrm{~kg}$ with the HR 1.52 . Weight gain didn't predict increased risk in our study. In women, U-shaped association was found while the HRs were 1.64 and 1.35 for those with $10 \mathrm{~kg}$ weight loss and weight gain, respectively. The risks for coronary and stroke mortality were also increased for women who lost their weight with the HRs 2.03 and 1.49. The risk trends were similar in subgroup analysis of elderly, non-smokers and early death deletion. Conclusion Weight loss since early adulthood was a predictor of death from CVD. Weight gain more than $10 \mathrm{~kg}$ was associated with increased risk of CVD mortality among women.

\section{P1-115 MEASURING THE HEALTH DISPARITY OF OLDER WOMEN IN SOUTH KOREA: USING MIXED METHODS}

doi:10.1136/jech.2011.142976d.9

H Chun, ${ }^{*}$ M Kang. Ewha Womans University, Seoul, Republic of Korea

Introduction Measuring appropriate socioeconomic positions (SEP) to capture health inequality among older women has always been a challenging issue. This study sought to explore mixed methods, applicable for measuring the health disparity of Korean women.

Methods We followed a sequential explanatory design, in which two data collection methods - qualitative and quantitative were employed to understand and identify appropriate ways to measure SEPs of older Korean women. In the multi-methods analysis stage, we used a concurrent triangulation design strategy and illustrated the major findings, according to 'corroboration', 'elaboration', and 'initiation' steps.

Results Most elder women reported their health to be relatively poorer in both quan/qual data, regardless of physician's report or diagnosis. Stress related mental problems were also found to be a major precursor to poorer physical health in these women. According to the findings, low education, financial problems, physically demanding work experience, and lack of social support resulted in poorer health. This was further supplemented by insightful explanations through in-depth interview. We further found cultural specific reasons behind this phenomenon particularly in regards to the education of and occupations of Korean women. They were often found to have been deprived of educational opportunities regardless of familial affluence. The educational levels of their male siblings, rather than their own, may better reflect the familial economic condition in the childhood of these Korean older women.

Conclusions The mixed methods, using both quantitative and qualitative approaches, could go some way towards explaining the health disparity in Korean older women.

\section{P1-116 ALCOHOL USE AMONG BRAZILIAN PHARMACY STUDENTS}

doi:10.1136/jech.2011.142976d.10

J Quintanilha, N Dewulf, H Coelho. ${ }^{*}$ Faculty of Pharmaceutical Sciences of Ribeirão Preto, University of São Paulo, Ribeirão Preto, São Paulo, Brazil

Introduction The significantly use of alcohol by the population poses a serious public health threat. Alcohol deserves special attention for being socially accepted. Studies have been shown that levels of alcohol consumption among students and young people are particularly high. The objective was to analyse the prevalence of alcohol used by Brazilian pharmacy students and describe the pattern of their consumption.

Methods The sample included 329 undergraduate pharmacy students of Faculty of Pharmaceutical Sciences of Ribeirão Preto, University of São Paulo, Brazil who completed an anonymous, selfapplied questionnaire adapted of the developed one for Brazilian Center Information of Psychoactive Drugs from august to december of 2010. This study was approved by Research Ethics Committee of the institution.

Results A total of 329 students answered the questionnaire and signed a free informed consent form. Age ranged from 17 to 28 years old (mean 21.1). The majority of students $(97.3 \%)$ had used alcohol at least once in their life. Of those participants, $68.8 \%$ drink beer and 21.6 drink wine. The study indicated that $79.1 \%$ of the students had drunk alcohol in the last month and up to $60 \%$ of them consumed alcohol for 5 days. Almost $40 \%$ of the students had driven a car after alcohol consumption.

Conclusion This study presented a high consumption of alcohol in pharmacy students in agreement with other studies. Measures to discourage alcohol consumption before driving car should be implemented. Approaches to alter student perceptions have been demonstrated to be useful and should be carried out.

\section{P1-117 DETERMINANTS OF FOLATE CONCENTRATION IN CANADIAN WOMEN OF CHILDBEARING AGE}

doi:10.1136/jech.2011.142976d.11

${ }^{1,2} \mathrm{C}$ K Colapinto, ${ }^{* 3,4} \mathrm{D}$ L O'Connor, ${ }^{2} \mathrm{~L}$ Dubois, ${ }^{1,5} \mathrm{M}$ S Tremblay. ${ }^{1}$ Children's Hospital of Eastern Ontario Research Institute, Healthy Active Living Obesity Research Group, Ottawa, Ontario, Canada; ${ }^{2}$ University of Ottawa Institute of Population Health, Ottawa, Ontario, Canada; ${ }^{3}$ The Hospital for Sick Children, Toronto, Ontario, Canada; ${ }^{4}$ University of Toronto, Department of Nutritional Sciences, Faculty of Medicine, Toronto, Ontario, Canada; ${ }^{5}$ University of Ottawa, Department of Pediatrics, Faculty of Medicine, Ottawa, Ontario, Canada

Objectives One-fifth of Canadian women of childbearing age have a red blood cell (RBC) folate below concentrations considered optimal for maximal neural tube defect risk reduction $(<906 \mathrm{nmol} / \mathrm{l})$. This study explored the determinants of $\mathrm{RBC}$ folate concentrations $\geq 906 \mathrm{nmol} / \mathrm{l}$ Canadian women.

Methods RBC folate concentrations from the nationally representative Canadian Health Measures Survey were assessed in women aged 15 to 45 years $(\mathrm{N}=1162)$. The population was described using frequencies and percentages. Sociodemographic, behavioural and clinical determinants of concentrations $\geq 906 \mathrm{nmol} /$ 1 were examined using univariate and separate multiple logistic regression models controlling for age and household income.

Results More women with folate concentrations $\geq 906 \mathrm{nmol} / 1$ were evident in higher age groups (15 to 19 years (70.0\%), 20 to 39 years $(79.6 \%), 40$ to 45 years $(80.8 \%))$ and income quartiles $(74.7 \%$ (Q1), 76.8\% (Q2), 80.2\% (Q3), 86.3\% (Q4)). Folic acid containing supplement users had a higher prevalence of concentrations $\geq 906 \mathrm{nmol} / \mathrm{l}$ (87.9\%) than non- users (75.2\%). Prevalence of concentrations $\geq 906 \mathrm{nmol} / 1$ rose with higher intake of fruit/vegetables ( $<$ once per day (64.7\%), 1-2 times per day $(74.2 \%)$ and $>3$ times per day $(82.9 \%)$ ) and grain products ( $<$ once per day $(67.7 \%)$, $1-2$ times per day $(80.5 \%),>3$ times per day $(77.6 \%))$. Detailed regression analysis results will be presented at the conference.

Interpretation These results will inform nutrition policies, particularly those related to folic acid fortification of select grains and preconception/prenatal folic acid supplementation recommendations for women of childbearing age. This will ultimately contribute to improving the health of Canadians and optimising infant health. 